\title{
Autotrophic Growth with Hydrogen of Mycobacterium gordonae and Another Scotochromogenic Mycobacterium
}

\author{
SANG S. PARK and B. T. DeCICCO
}

Department of Biology, The Catholic University of America, Washington, D.C. 20017

\begin{abstract}
An acid-fast, slow-growing scotochromogenic mycobacterium was isolated from a medium specific for hydrogen-utilizing chemolithotrophs. The organism grew well in pure culture in simple mineral salts media under an atmosphere of hydrogen, oxygen, and carbon dioxide. No growth occurred in the absence of the gas mixture unless organic substrates were added. Four tested strains of the tap-water scotochromogen, Mycobacterium gordonae, were also able to grow autotrophically, whereas none of eight tested strains of $M$. scrofulaceum grew using hydrogen. Twenty-one other mycobacterial strains were negative for autotrophic growth; a strain of $M$. xenopi grew very slowly. The isolated scotochromogen conformed to the properties of $M$. scrofulaceum except for its ability to grow at $19 \mathrm{C}$ and its autotrophic ability. This organism exhibited two major colony types. During autotrophic cultivation, a flat, rough colony form was dominant; heterotrophic cultivation caused a population shift to a smooth, domed variety. The two colony forms exhibited qualitatively similar biochemical properties, and the unusual rough-to-smooth transition seemed to correlate with the quantitatively enhanced heterotrophic growth capacities of the smooth strain. Rough-to-smooth variation was reversible, and predominantly smooth inocula gave rise to predominantly rough populations under conditions of chemoautotrophy. The ability to grow autotrophically may be a useful characteristic for distinguishing the saprophytic scotochromogens from the more pathogenic strains.
\end{abstract}

A number of reports $(2,5,10)$ indicate the ability of some members of the order Actinomycetales to grow autotrophically in mineral salts media under an atmosphere of hydrogen, oxygen, and carbon dioxide. Some early studies reporting autotrophic growth of mycobacteria attempted to correlate this ability with hydrocarbon utilization, and the strains of mycobacteria were generally reported to be rapid-growing soil and water saprophytes $(2,10)$. One study listed a "tap water" type among some mycobacterial strains found to grow autotrophically (10); other strains were identified as members of the genus Nocardia (5). However, we are not aware of any detailed investigation of autotrophic capacities among the slowgrowing mycobacteria. In addition, there have been no reports suggesting the importance of the chemolithotrophic capacity for distinguishing the normally saprophytic tap-water scotochromogen Mycobacterium gordonae from the similar but more commonly pathogenic $M$. scrofulaceum and from other mycobacteria (12).

The chemolithotrophic capacity of $M$. gordonae and a new scotochromogenic isolate and the results of some physiological studies with rough and smooth variants of the isolated mycobacterium are included in this report.

\section{MATERIALS AND METHODS}

Bacterial strains. The mycobacterial strains employed in the present study and their sources are listed in Table 1. Strains AU and $6 \mathrm{Y}$ are rough and smooth variants, respectively, of the mycobacterium originally isolated as a contaminant in an autotrophic culture.

Media and growth conditions. The chemolithotrophic growth medium consisted of $0.1 \%\left(\mathrm{NH}_{4}\right)_{2}$ $\mathrm{SO}_{4}, 0.02 \% \mathrm{MgSO}_{4} \cdot 7 \mathrm{H}_{2} \mathrm{O}$, and $0.001 \% \mathrm{Fe}\left(\mathrm{NH}_{4}\right)_{2}$ $\left(\mathrm{SO}_{4}\right)_{2} \cdot 6 \mathrm{H}_{2} \mathrm{O}$ in $0.04 \mathrm{M}$ potassium phosphate buffer, pH 6.7. The ferrous salt was prepared as a concentrated stock solution in deionized water (adjusted to pH 3.0 with $\mathrm{HCl}$ ) and was sterilized with a $0.45-\mu \mathrm{m}$ membrane filter (Millipore Corp., Bedford, Mass.). It was added to the autoclaved and cooled mineral salts solution to the designated concentration. For autotrophic growth, 250-ml Erlenmeyer flasks containing $100 \mathrm{ml}$ of mineral salts medium were inoculated and incubated under an atmosphere of $50 \% \mathrm{H}_{2}, 10 \% \mathrm{CO}_{2}$, and $40 \%$ air or $50 \% \mathrm{H}_{2}, 10 \% \mathrm{CO}_{2}, 20 \% \mathrm{O}_{2}$, and $20 \%$ $\mathrm{N}_{2}$. For autotrophic cultivation on solid media, $0.85 \%$ Ionagar no. 2 (Colab) was added to the mineral salts medium. The gas mixture was continuously 
TABLE 1. Some differential characters of mycobacterial strains

\begin{tabular}{|c|c|c|c|c|c|c|c|}
\hline \multirow[b]{2}{*}{ Species } & \multirow[b]{2}{*}{ Strains tested ${ }^{a}$} & \multicolumn{3}{|c|}{ Growth at } & \multicolumn{2}{|c|}{$\begin{array}{l}\text { Tween } 80 \\
\text { hydrolysis }\end{array}$} & \multirow{2}{*}{$\begin{array}{l}\text { Chemolithotro- } \\
\text { phic growth at } \\
35 \mathrm{C}\end{array}$} \\
\hline & & $19 \mathrm{C}$ & $35 \mathrm{C}$ & $45 \mathrm{C}$ & 5 days & 10 days & \\
\hline M. tuberculosis & E01, E07 & & + & & - & - & $-b$ \\
\hline M. kansasii & $\begin{array}{l}\text { E06, TMC, } 1201,1204, \\
\quad 1214,1217\end{array}$ & & + & & + & + & \\
\hline M. marinum & E08, TMC, 1218,1219 & & + & & + & + & - \\
\hline M. gordonae & $\begin{array}{l}\mathrm{E} 02, \mathrm{TMC}, 1318,1319 \\
1324\end{array}$ & + & + & - & + & + & $+^{c}$ \\
\hline Mycobacterium sp. & $\mathrm{AU}, 6 \mathrm{Y}$ & + & + & - & - & - & + \\
\hline M. scrofulaceum & $\begin{array}{c}\text { E03, TMC, } 1302,1306 \\
1314,1316,1323\end{array}$ & _- & + & - & - & - & - \\
\hline & TMC 1305,1307 & \pm & + & - & - & - & - \\
\hline M. flavescens & TMC 1541 & + & + & - & + & + & - \\
\hline M. avium & TMC 706 & & + & & - & - & - \\
\hline M. intracellutare & TMC 1406 & & + & & - & - & - \\
\hline M. nonchromo- & & & & & & & \\
\hline genicum & TMC 1459 & & + & & + & + & - \\
\hline M. xenopi & TMC 1470 & - & + & + & - & - & $-^{d}$ \\
\hline M. smegmatis & TMC 1546 & & + & & + & + & - \\
\hline M. phlei & TMC 1516,1523 , & & & & & & \\
\hline & ATCC 354 & & + & & - & - & - \\
\hline M. fortuitum & TMC 1529 & & + & & \pm & \pm & - \\
\hline M. diernhoferi & TMC 1540 & & + & & - & - & - \\
\hline M. chelonei & TMC 1542 & & + & & - & - & - \\
\hline
\end{tabular}

${ }^{a}$ Strains with E designations are from the College of American Pathologists. TMC, Trudeau Mycobacterial Culture Collection. ATCC, American Type Culture Collection, Rockville, Md.

$b$ No growth within 45 days.

c Growth within 7 days.

$d$ Some growth at 29 days.

supplied to the culture flasks or vacuum jars through a manifold from a gas reservoir and was maintained at slightly greater than atmospheric pressure as previously described (1). For heterotrophic growth in liquid media, Middlebrook 7H9 broth (Difco) enriched with oleic acid, bovine albumin fraction V, D-glucose, beef catalase, sodium chloride (OADC, Difco), and $0.2 \%$ glycerol (Difco) was employed.

Cells from Middlebrook 7H10 agar slants (enriched with glycerol and OADC as described by Difco) were inoculated into enriched $7 \mathrm{H} 9$ broth and grown for 2 to 3 days at $35 \mathrm{C}$ during which time the turbidity increased 3- to 4-fold. These broth cultures were then used to inoculate tubes containing $10 \mathrm{ml}$ of enriched $7 \mathrm{H} 9$ broth to an optical density at $540 \mathrm{~nm}\left(\mathrm{OD}_{540}\right)$ of 0.05 . Growth was monitored by following the increase in turbidity at $540 \mathrm{~nm}$, using a Spectronic 20 colorimeter (Bausch and Lomb). An $\mathrm{OD}_{540}$ of 0.3 corresponded to $2.1 \times 10^{8}$ colony-forming units (CFU) $/ \mathrm{ml}$ and a cellular dry weight of $0.13 \mathrm{mg} / \mathrm{ml}$ for heterotrophically grown cells $(7 \mathrm{H} 9$ broth) and $3.7 \times$ $10^{7} \mathrm{CFU} / \mathrm{ml}$ and $0.18 \mathrm{mg}$ (dry weight) $/ \mathrm{ml}$ for autotrophically grown cells. The wide disparity in the CFU and dry weight values of heterotrophic and autotrophic cultures is probably explained by the granular nature of the autotrophic broth cultures (causing higher cell mass and lower CFU per OD unit than the smooth heterotrophic cells).
For maintenance of cultures, plate counts, and observation of colony morphology and pigmentation, enriched $7 \mathrm{H} 10$ agar was generally employed. In designated instances, Lowenstein-Jensen medium (Difco) or Micro Inoculum broth (Difco) was employed.

Tests for utilization of organic substrates. Inocula were prepared by growing cells in $7 \mathrm{H} 9$ broth at $35 \mathrm{C}$ for 2 to 3 days, washing by centrifugation, and resuspending in sterile mineral salts medium without iron. A single organic compound, autoclaved separately in deionized water, was added to mineral salts medium to the designated concentration. The inoculated tubes were incubated at $35 \mathrm{C}$ with shaking. During growth experiments, all cultures were checked for contamination by streaking on tryptic soy agar (Difco) plates and by acid-fast staining.

Biochemical tests and serology. The production of niacin was determined by TB niacin test strips (Difco). Nitrate reduction was detected by growing cells in indole-nitrate broth (Difco), acidifying the culture with one drop of a $1: 1$ dilution of concentrated $\mathrm{HCl}$, and adding two drops of $0.2 \%$ sulfanilamide and $0.1 \%$ $\alpha$-naphthylamine. This method yielded results identical to those reported using the standard mycobacterial nitrate reduction test (8). Control strains known to possess nitrate-reducing ability produced positive reactions after 2 to $4 \mathrm{~h}$ of incubation, whereas known 
negative strains gave negative reactions even after $24 \mathrm{~h}$ of incubation in the indole-nitrate medium. Catalase activity $(17,18)$ and Tween 80 hydrolysis (16) were tested according to the methods of Wayne, and urease activity was determined according to the method of Singer and Cysner (15) by using cells grown on 7H10 agar slants. Tellurite reduction was determined by adding two drops of a sterile $0.2 \%$ solution of potassium tellurite to a 7 -day-old culture in $7 \mathrm{H} 9$ broth and incubating at $35 \mathrm{C} \mathrm{(8)}$.

Serotyping of strains $\mathrm{AU}$ and $6 \mathrm{Y}$ was performed by E. Wolinsky and W. B. Schaefer using the procedures of Schaefer (13).

Effect of pH on growth. The same mineral salts medium used for autotrophic grow th was employed to determine the effect of $\mathrm{pH}$ on autotrophic growth. The $0.04 \mathrm{M}$ potassium phosphate buffer used as the mineral medium base was adjusted to produce media with initial $\mathrm{pH}$ values between 5.5 and 8.5 . Cells were grown autotrophically at room temperature for 17 days, harvested by centrifugation, resuspended in mineral salts medium without iron ( $\mathrm{pH} 6.7$ ), and used as inocula. For each $\mathrm{pH}$ tested between 5.5 and 8.5 , $100 \mathrm{ml}$ of mineral salts medium in a $250-\mathrm{ml}$ Erlenmeyer flask was inoculated to an OD (calculated) of 0.006 and incubated at room temperature under an atmosphere of $50 \% \mathrm{H}_{2}, 10 \% \mathrm{CO}_{2}, 20 \% \mathrm{O}_{2}$, and $20 \%$ $\mathrm{N}_{2}$.

\section{RESULTS}

Isolation of rough and smooth strains. Strains $\mathrm{AU}$ and $6 \mathrm{Y}$ were derived from a single contaminating organism in a 2 -month-old continuous culture of a nonpigmented, hydrogenutilizing chemolithotrophic bacterium. The organism was noticed as an orange-pigmented pellicle attached to the gas sparger just above the liquid surface and was purified by repeated cloning onto tryptic soy agar.

The newly isolated organism grew slowly on $7 \mathrm{H10}$ agar, Micro Inoculum agar (made by adding $15 \mathrm{~g}$ of agar [Difco] per liter to Micro Inoculum broth), or Lowenstein-Jensen egg medium at $35 \mathrm{C}$. It formed barely visible colonies in 4 to 5 days and large colonies in 2 to 3 weeks. The organism was strongly acid fast and was identified as a yellow scotochromogen (Table 2).

Originally the isolated strain formed rough colonies on autotrophic agar or tryptic soy agar, but after prolonged incubation on certain heterotrophic media, especially Micro Inoculum agar or enriched $7 \mathrm{H} 10$ agar supplemented with $0.1 \%$ aspartic acid, smooth, more intensely pigmented colonies grew out of the rough colonies. Both colony types were capable of autotrophic growth. Since the rough culture grew better than the smooth isolates under chemolithotrophic conditions, the original
TABLE 2. Some characteristics of rough $(A U)$ and smooth (6Y) strains of an isolated scotochromogenic mycobacterium

\begin{tabular}{|c|c|c|}
\hline \multirow[b]{2}{*}{ Character } & \multicolumn{2}{|c|}{ Strain } \\
\hline & $\mathrm{AU}$ & $6 \mathrm{Y}$ \\
\hline Growth rate & Slow & Slow \\
\hline Growth at: & & \\
\hline $19 \mathrm{C}$ & + & + \\
\hline $35 \mathrm{C}$ & + & + \\
\hline $45 \mathrm{C}$ & - & - \\
\hline Niacin & - & - \\
\hline Nitrate reduction & - & - \\
\hline Catalase activity: & & \\
\hline $45 \mathrm{~mm}$ foam & + & + \\
\hline $68 \mathrm{C} / 20 \mathrm{~min}$ & + & + \\
\hline Pigment: & & \\
\hline dark & $\mathrm{OY}^{a}$ & OY \\
\hline light & OY & OY \\
\hline Tween 80 hydrolysis: & & \\
\hline 5 days & - & - \\
\hline 10 days & - & - \\
\hline Tellurite reduction: 3 days & - & - \\
\hline Urease activity & + & + \\
\hline Chemolithotrophic growth & + & + \\
\hline
\end{tabular}

${ }^{a} \mathrm{OY}$, Light orange/yellow.

rough strain was designated as strain $\mathrm{AU}$ (for autotrophic), and one of the smooth clones was designated $6 \mathrm{Y}$.

When strain AU was grown autotrophically and then spread onto $7 \mathrm{H} 10$ agar plates and incubated at $35 \mathrm{C}$ for 25 days, the great majority of colonies were rough, flat, and light yellow in color. A small number of smooth, domed colony types usually were observed growing out of the rough colonies, and these papillae continued to grow until they were much larger than the surrounding rough types. The occurrence of smooth variants seemed to be a function of the richness of the medium. Autotrophic cultures spread on media which did not support luxurious growth, such as tryptic soy agar, did not give rise to significant numbers of smooth colonies. Cultures on $7 \mathrm{H} 10$ agar or Micro Inoculum agar yielded larger numbers of outgrowing smooth colonies. This suggests that the smooth colony variants are due to mutation and that the rough parent strain has to attain large populations on 
heterotrophic agar betore smooth mutants are observed. The production of variants occurred in both directions; strain $6 \mathrm{Y}$ grown heterotrophically and spread on 7H10 agar yielded a very small proportion of rough colony types in addition to the normal smooth colonies. Repeated cloning of $\mathrm{AU}$ and $6 \mathrm{Y}$ cultures to ensure purity did not reduce their ability to undergo variation.

Characteristics of strains $\mathrm{AU}$ and $6 \mathrm{Y}$. The reactions of strains $\mathrm{AU}$ and $6 \mathrm{Y}$ to some of the key differentiating tests used for the identification of mycobacterial strains $(7,19,20)$ are shown in Table 2. Both rough (AU) and smooth (6Y) types were identical with respect to those characters tested. Growth as observed on $7 \mathrm{H} 10$ agar slants and plates wrapped with aluminum foil and incubated at $35 \mathrm{C}$ revealed both strains to be slow-growing scotochromogens, strongly catalase positive, but negative for hydrolysis of Tween 80. Both AU and $6 \mathrm{Y}$ grew autotrophically in liquid media and on mineral salts Ionagar plates under the autotrophic gas mixture. Neither strain produced niacin, and both strains were unable to reduce nitrate and potassium tellurite.

Utilization of organic substrates. Since heterotrophic media seemed to favor the proliferation of the smooth strain, the two strains were tested for their capacity to grow by utilizing a number of organic substances as single sources of carbon and energy. The results revealed no qualitative distinctions between the strains, but some quantitative differences were observed (Table 3). Both strains could utilize glucose,

TABLE 3. Utilization of substrates as carbon and energy sources by strains $A U$ and $6 Y$

\begin{tabular}{l|c|c||l|c|c}
\hline \multirow{2}{*}{ Substrate } & \multicolumn{2}{|c||}{ Strain } & & \multicolumn{2}{c}{ Strain } \\
\cline { 2 - 3 } & AU & $6 \mathrm{Y}$ & Substrate & AU & $6 \mathrm{Y}$ \\
\hline Arabinose & $-b$ & - & Glutamine & - & - \\
Galactose & - & - & Glycerol & + & $6+$ \\
Glucose & + & $4+^{c}$ & Propionic acid & + & + \\
Lactose & - & - & Caproic acid & - & - \\
Citrate & - & - & Caprylic acid & + & + \\
Aspartate & - & - & Capric acid & + & + \\
Asparagine & - & - & Palmitic acid & + & $2+$ \\
Glutamate & - & - & Oleic acid & + & $2+$ \\
\hline
\end{tabular}

${ }^{a}$ All substrates were in mineral salts medium at a concentration of $0.1 \%$ except for propionic acid, which was at a concentration of $0.01 \%$.

$b$ All tubes were initially inoculated to an $\mathrm{OD}_{540}$ of $0.05 ;(-)$ indicates no increase in turbidity within 30 days.

${ }^{c}$ The numbers before the $(+)$ signs indicate the relative amount of growth compared with the $\mathrm{AU}$ culture using the same substrate. glycerol, propionate, caprylate, caprate, palmitate, and oleate, but strain $6 \mathrm{Y}$ grew much better on glucose and glycerol and somewhat better on palmitate and oleate than did strain AU. No growth was observed with either strain in tubes containing arabinose, galactose, lactose, citrate, aspartate, asparagine, glutamate, glutamine, or caproate.

Heterotrophic growth. The growth patterns for strains $\mathrm{AU}$ and $6 \mathrm{Y}$ in $7 \mathrm{H} 9$ broth at $35 \mathrm{C}$ with shaking are shown in Fig. 1. Growth under these conditions was superior to growth at room temperature or $30 \mathrm{C}$ with shaking or at any of the three temperatures without shaking. The doubling times for the initial, rapid grow th phase were $6.6 \mathrm{~h}$ for strain $\mathrm{AU}$ and $6.9 \mathrm{~h}$ for strain 6Y; however, growth rapidly slowed to yield mean generation times over the first $24-\mathrm{h}$ period of approximately $10.7 \mathrm{~h}$ for both strains. The growth of AU always leveled off at lower cell densities than did $6 \mathrm{Y}$, supporting similar observations on heterotrophic agar plates as mentioned above. Heterotrophic growth of $M$. gordonae EO2 closely paralleled that of $6 \mathrm{Y}$.

Chemolithotrophic growth in liquid media. When inoculated directly from a 7H10 agar slant into autotrophic media ( $\mathrm{pH}$ 6.7), both strain AU and strain $6 \mathrm{Y}$ experienced a 5- to 6-day lag before significant growth occurred. When inoculation was from an autotrophically

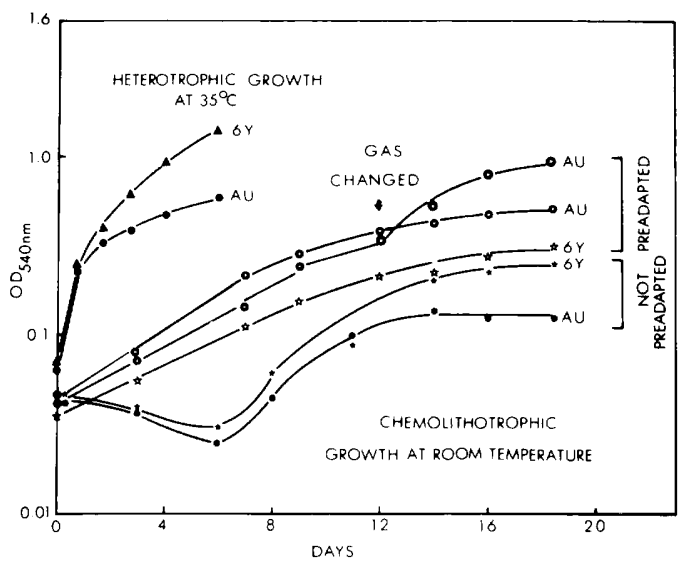

FIG. 1. Growth of Mycobacterium sp. strains $A U$ and $6 Y$ under heterotrophic and chemolithotrophic conditions. Heterotrophic cultivation was in $7 \mathrm{H} 9$ broth. For autotrophic growth cells grown on $7 \mathrm{HIO}$ agar or autotrophically, pregrown cells were inoculated into mineral salts media and incubated under an atmosphere of $50 \% \mathrm{H}_{2}, 10 \% \mathrm{CO}_{2}$, and $40 \%$ air. At the indicated time the gas mixture in one of the preadapted $\mathrm{AU}$ cultures was changed to $50 \% \mathrm{H}_{2}, 10 \%$ $\mathrm{CO}_{2}, 20 \% \mathrm{O}_{2}$, and $20 \% \mathrm{~N}_{2}$, producing the observed stimulation of growth. 
grown culture, exponential growth began without a lag (Fig. 1). The generation times during the initial period of growth by the preadapted cells were 3.3 days for strain $\mathrm{AU}$ and 4.9 days for strain $6 \mathrm{Y}$. A sudden increase in growth was observed when the oxygen concentration was increased from 8 to $20 \%$, suggesting that oxygen became limiting in our system at an OD of approximately 0.3 to 0.5 , the cell density at which exponential growth ended.

Effect of $\mathrm{pH}$ on chemolithotrophic growth. When strain AU was inoculated into mineral salts media of different $\mathrm{pH}$ values and grown under autotrophic conditions, optimal growth was obtained with media containing an initial $\mathrm{pH}$ of 7.0 after 15 days and with a medium at pH 6.5 after 25 days (Fig. 2). The initial pH values decreased by as much as 0.35 to $0.45 \mathrm{U}$ during the 25-day incubation. The size of the inoculum had no significant effect on the optimal $\mathrm{pH}$ for growth. We therefore concluded that the pH 6.7 mineral salts medium which we were using was near optimum for autotrophic growth studies.

Chemolithotrophic growth on solid media. Since strains $\mathrm{AU}$ and $6 \mathrm{Y}$ were identified as scotochromogens, we tested a number of other strains of mycobacteria, both scotochromogens

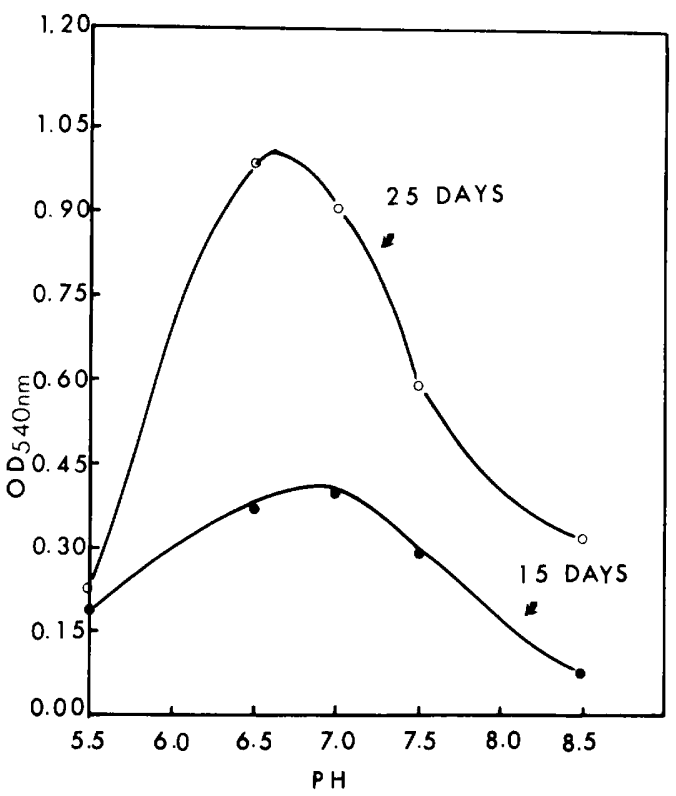

FIG. 2. Effect of $p H$ on the chemolithotrophic growth of strain AU. Autotrophically grown cells were inoculated into 250-ml Erlenmeyer flasks containing $100 \mathrm{ml}$ of mineral salts media at different $\mathrm{pH}$ values and incubated under an atmosphere of $50 \% \mathrm{H}_{2}, 10 \%$ $\mathrm{CO}_{2}, 20 \% \mathrm{O}_{2}$, and $20 \% \mathrm{~N}_{2}$ at room temperature with shaking. The initial $O D_{540}$ was 0.05 . and non-scotochromogens, for chemolithotrophic capacity. For these studies we first determined that strains $\mathrm{AU}$ and $6 \mathrm{Y}$ exhibited luxurious growth when streaked onto mineral salts-Ionagar plates and incubated in a vacuum jar under an atmosphere of $50 \% \mathrm{H}_{2}, 10 \% \mathrm{CO}_{2}$, and $40 \%$ air. Growth could be observed on plates incubated at $35 \mathrm{C}$ in 4 to 5 days and was heavy by 20 days. No growth was observed if the gas atmosphere was replaced by air, demonstrating that there was not sufficient contaminating organic matter to support visible growth. In addition to strains $\mathrm{AU}$ and $6 \mathrm{Y}, 34$ other mycobacterial strains were tested on solid media for their capacity to grow autotrophically with hydrogen at $35 \mathrm{C}$ and at room temperature (Table 1). The four strains listed as $M$. gordonae, including the three strains of this species obtained from the Trudeau Mycobacterial Collection, grew well autotrophically. None of the other strains tested showed this capacity, including strains of $M$. tuberculosis, $M$. kansasii, $M$. marinum, $M$. flavescens, $M$. scrofulaceum, $M$. avium, $M$. intracellulare, $M$. nonchromogenicum, $M$. smegmatis, $M$. phlei, $M$. fortuitum, $M$. diernhoferi, and $M$. chelonei. The one strain of $M$. xenopi that was tested showed slight growth on the mineral agar after 29 days and thus seemed capable of very slow autotrophic growth. However, this organism was distinguishable from the other autotrophic strains by its ability to grow at $45 \mathrm{C}$ (Table 1).

Results with some selected characters revealed that strains $\mathrm{AU}$ and $6 \mathrm{Y}$ resemble $M$. gordonae and differ from $M$. scrofulaceum in their ability to grow at $19 \mathrm{C}$. However, strains $\mathrm{AU}$ and $6 \mathrm{Y}$ resemble $M$. scrofulaceum in their inability to hydrolyze Tween 80 in 10 days (Table 1) and their positive urease test (Table 2). Also, serological studies revealed that both strains were agglutinated by $M$. scrofulaceum antisera.

Figure 3 shows the autotrophic growth of strains $\mathrm{AU}$ and $6 \mathrm{Y}$ and the four strains of $M$. gordonae on agar plates. Six of the eight tested strains of $M$. scrofulaceum were inoculated on this same plate in the areas between strains AU and $6 \mathrm{Y}$ and the $M$. gordonae strains to demonstrate the distinctive nature of this test. The photograph was taken after 20 days of incubation at $35 \mathrm{C}$.

\section{DISCUSSION}

The important role medium composition plays in the colony morphology of mycobacteria and the variations in biochemical activity 


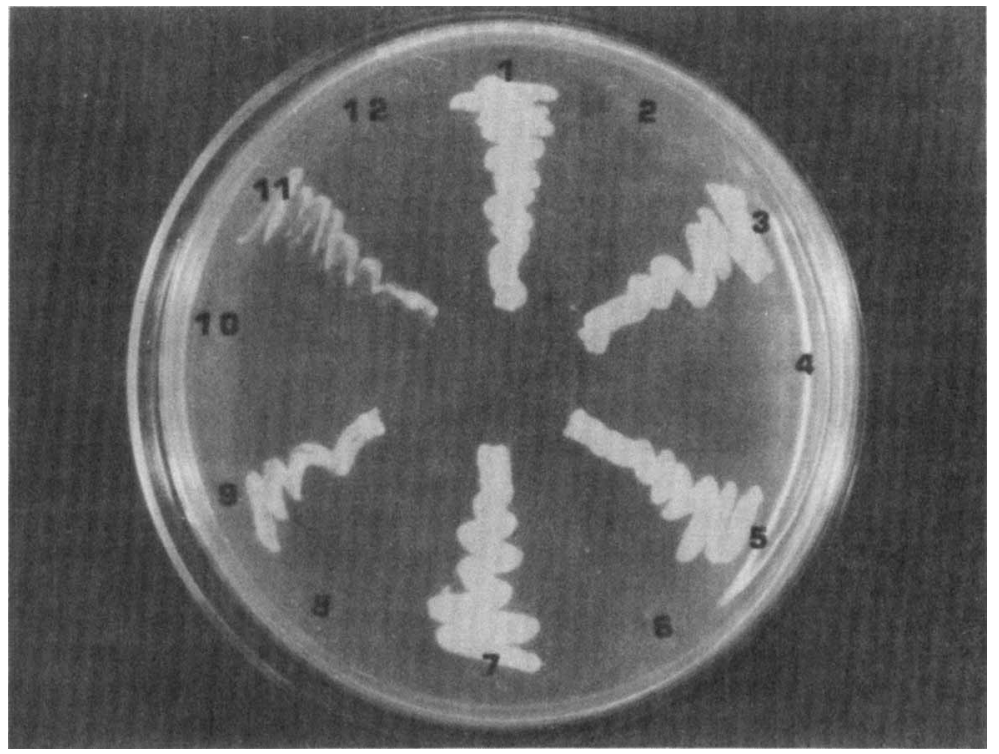

FIG. 3. Autotrophic growth of mycobacterial strains on mineral salts agar. Incubation was for 20 days at $35 \mathrm{C}$ under an atmosphere of $50 \% \mathrm{H}_{2}, 10 \% \mathrm{CO}_{2}, 20 \% \mathrm{O}_{2}$, and $20 \% \mathrm{~N}_{2}, 1,3,5,7,9,11: \mathrm{M}$. gordonae strains TMC1318, TMC1319, TMC1324, EO2, strain $A U$, and strain $6 Y$, respectively. 2, 4, 6, 8, 10 and $12: M$. scrofulaceum strains TMC1302, TMC1305, TMC1306, TMC1307, TMC1314, and EO3, respectively.

of rough and smooth mycobacteria have previously been reported. $(3,9)$. Our observations on the stimulation of the autotrophic growth rate by increased oxygen partial pressures and the need for adequate aeration to maximize growth in liquid media resemble the oxygen effect on mycobacterial growth as noted by Halpern and Kirchheimer (4). The outgrowth of smooth colony forms from rough cultures is apparently quite unusual and seems to be due to some nutrient limiting the growth of the rough organisms, since the rough colonies cease growing long before the smooth colonies reach full size. The superior capacity of smooth organisms to use some unidentified substrate may be responsible for this effect, or the ability of smooth forms to grow better in low oxygen pressures may be a factor. We suspect that oxygen is limiting during cultivation in liquid media because of the growth rates seen in Fig. 1. For example, doubling times of 3 to 5 days during autotrophic growth cannot account for the appearance of tiny colonies on autotrophic agar after only 12 to 15 days. Assuming that these colonies developed from only one or a small number of cells, we estimate that a doubling time of 12 to $20 \mathrm{~h}$ would be necessary. The availability of oxygen to surface cultures on agar plates may explain the difference.

A cooperative numerical taxonomic analysis of the slow-growing scotochromogenic mycobacteria (Runyon's group II) revealed four clusters (20). Three of these conformed to named species ( $M$. flavescens, $M$. gordonae, and $M$. scrofulaceum). It is suggested that a fourth cluster consisting of four strains may comprise a new species. Twelve characters seemed to differentiate most strains of $M$. gordonae from $M$. scrofulaceum. Of these, Tween hydrolysis and phosphatase activity were most definitive, Tween hydrolysis being the only character that differentiated all strains of both species (20).

Strains $\mathrm{AU}$ and $6 \mathrm{Y}$ were negative for Tween hydrolysis and positive for urease; these results place these strains with $M$. scrofulaceum $(7,19$, $21,22)$. However, their ability to grow at $19 \mathrm{C}$ is a character associated with M. gordonae. The chemolithotrophic capacity was definitive in differentiating four strains of $M$. gordonae from eight strains of $M$. scrofulaceum. Based on the four characters mentioned (urease, Tween hydrolysis, growth at $19 \mathrm{C}$, chemolithotrophy), strains $\mathrm{AU}$ and $6 \mathrm{Y}$ would seem to be intermediate between these two species. Yet they do not fit the intermediate cluster of Wayne et al. (20) because of their inability to hydrolyze Tween 80 and their positive urease reaction. Their origin as air contaminants suggests that they are soil or water saprophytes, as commonly is the case with $M$. gordonae. Growth at $19 \mathrm{C}$ also seems to be a trait more associated 
with saprophytic types, whereas the opposite is generally true for clinically important organisms. The serotype numbering scheme proposed by Wolinsky and Schaefer, which is based on serological agglutination (23), would eliminate the necessity for a species name in favor of an arabic number under the heading " $M$. avium Complex." According to this scheme, the isolated organism is related to $M$. scrofulaceum and belongs to sereotype 41 (E. Wolinsky, personal communication).

Autotrophic microorganisms are generally isolated from soil and water where this ability may play a role in allowing these organisms to exist under diverse environmental conditions. While one cannot generalize on the basis of a single strain, the yellow pigmentation and (slow) autotrophic growth of $M$. xenopi may indicate that this organism bears some relationship to the autotrophic, saprophytic scotoch romogens described in this report. One intriguing possible exception is the recent suggestion that $M$. leprae might grow chemoautotrophically during its intracellular existence (6), but this conclusion, based on the presence of high levels of ribulose-diphosphate carboxylase and little or no reduced nicotinamide adenine dinucleotide oxidase and 2-oxoglutarate dehydrogenase, needs to be confirmed.

We suggest that chemolithotrophic growth may be a useful character for differentiating saprophytic from potentially pathogenic or other clinically significant strains of scotochromogenic mycobacteria $(14,20,22)$. Whether this distinction will remain highly significant will depend upon the results of more extensive studies. In this regard, two saprophytic scotochromogenic mycobacteria isolated in Japan and listed as M. cuneatum (ATCC 21498) and $M$. petroleophilum (ATCC 21497) are reported to grow autotrophically (E. H. Runyon, personal communication). The final resolution of the autotrophic mycobacteria and their relationship to the presently established taxa must await further genetic, biochemical, and serological $(11,13,20)$ studies.

\section{ACKNOWLEDGMENTS}

We thank Ernest H. Runyon for his valuable suggestions concerning this manuscript. We also thank George P. Kubica for cultures from the Trudeau Mycobacterial Collection. The serological studies were kindly performed by Emanuel Wolinsky and Werner B. Schaefer.

Some materials used in this study were provided by the U.S.-Japan Cooperative Medical Science ProgramNIAID. These studies were supported in part by NASA grants NGR 09-005-022 and NGR 09-005-098.

\section{REPRINT REQUESTS}

Address reprint requests to: B. T. DeCicco, Dept. of Biology, Catholic University, Washington, D.C. 20017.

\section{LITERATURE CITED}

1. DeCicco, B. T., and P. E. Stukus. 1968. Autotrophic and heterotrophic metabolism of Hydrogenomonas. I. Growth yields and patterns under dual substrate conditions. J. Bacteriol. 95:1469-1475.

2. Dworkin, M., and J. W. Foster. 1958. Experiments with some microorganisms which utilize ethane and hydrogen. J. Bacteriol. 75:592-603.

3. Fregnan, G. B., and D. W. Smith. 1962. Description of various colony forms of mycobacteria. J. Bacteriol. 83:819-827.

4. Halpern, B., and W. F. Kirchheimer. 1954. Studies on the growth of mycobacteria. II. The effect of oxygenation and aeration on growth patterns of my cobacteria. Amer. Rev. Tuberc. 70:665-671.

5. Hirsch, P. 1961. Wasserstoffactivierung und Chemoautotrophie bei Actinomyceten. Archiv. Mikrobiol. 39:360-373.

6. Kato, L., D. Ajdukovic, A. Donawa, and M. Ishaque. 1973. Implication of chemolithotrophism in $M$. leprae. Nature N. Biol. 242:179-180.

7. Kubica, G. P. 1973. Differential identification of mycobacteria. VII. Key features for identification of clinically significant mycobacteria. Amer. Rev. Resp. Dis. 107:9-21.

8. Kubica, G. P., and W. E. Dye. 1967. Laboratory methods for clinical and public health mycobacteriology. Public Health Service Publication no. 1547. U.S. Government Printing Office, Washington, D.C.

9. Kubica, G. P., and W. D. Jones, Jr. 1970. The variation in biochemical reactivity of rough and smooth variants of mycobacteria. Pneumonologie 142:108-111.

10. Lukins, H. B., and J. W. Foster. 1963. Utilization of hydrocarbons and hydrogen by mycobacteria. Z. Allg. Mikrobiol. 3:251-264.

11. Reznikov, M., and J. H. Leggo. 1972. Modification of Schaefer's procedure for serotyping of organisms of the Mycobacterium avium $-M$. intracellulare-M. scrofulaceum complex. Appl. Microbiol. 23:819-823.

12. Runyon, E. H. 1959. Anonymous mycobactera in pulmonary disease. Med. Clin. N. Amer. 43:273-290.

13. Schaefer, W. B. 1965. Serological identification and classification of the atypical my cobacteria by their agglutination. Amer. Rev. Resp. Dis. 92:85-93.

14. Schaefer, W. B. 1968. Incidence of the serotypes of Mycobacterium avium and atypical mycobacteria in human and animal diseases. Amer. Rev. Resp. Dis. 97:18-23.

15. Singer, J., and E. Cysner. 1952. Urease activity in Mycobacteriaceae. Amer. Rev. Tuberc. 65:779-781.

16. Wayne, L. G. 1962. Differentiation of mycobac- 
teria by their effect on Tween 80. Amer. Rev. Resp. Dis. 86:579-581.

17. Wayne, L. G. 1962. Two varieties of Mycobacterium kansasii with different clinical significance. Amer. Rev. Resp. Dis. 86:651-656.

18. Wayne, L. G. 1966. Classification and identification of mycobacteria. Species within Group III. Amer. Rev. Resp. Dis. 93:919-928.

19. Wayne, L. G. 1970 . On the identity of Mycohacterium gordonae Bojalil and the so-called tap water scotochromogens. Int. J. Syst. Bacteriol. 28:149-153.

20. Wayne, L. G., T. M. Dietz, C. Gernez-Rieux, P. A. Jenkins, W. Käppler, G. P. Kubica, J. B. G. Kwapinski, G. Meissner, S. R. Pattyn, E. H.
Runyon, K. H. Schröder, V. A. Silcox, A. Tacquet, M. Tsukamura, and E. Wolinsky. 1971. A cooperative numerical analysis of scotochromogenic slowly growing mycobacteria. J. Gen. Microbiol. 66:255-271.

21. Wayne, L. G., J. R. Doubek, and G. A. Diaz. 1967. Classification and identification of mycobacteria. IV. Some important scotochromogens. Amer. Rev. Resp. Dis. 96:88-95.

22. Wolinsky, E. 1963. The role of scotochromogenic mycobacteria in human disease. Ann. N.Y. Acad. Sci. 106:67-71.

23. Wolinsky, E., and W. B. Schaefer. 1973. Proposed numbering scheme for mycobacterial serotypes by agglutination. Int. J. Syst. Bacteriol. 23:182-183. 